\title{
edmetic
}

Revista de Educación Mediática y TIC

Propuesta para un centro educativo 2.0

A porposal for school 2.0

Fecha de recepción: 10/05/2014

Fecha de revisión: 15/08/2015

Fecha de aceptación: 20/04/2016 
Propuesta para un centro educativo 2.0

A proposal for a school 2.0

\title{
Juan Carlos Araujo Portugal'
}

\begin{abstract}
Resumen:
En la actualidad el espíritu de la Web 2.0, por el que los usuarios de la red han dejado de ser meros consumidores de información para convertirse en productores de la misma de forma colaborativa, ha invadido todos los ámbitos de la vida cotidiana de las personas, y el contexto educativo no puede ser una excepción. En este artículo se presenta una propuesta para sacar partido a algunas herramientas que acompañan a la Web 2.0 para que todos los que tienen que ver con un centro educativo se sientan realmente involucrados y tengan la sensación de pertenecer a una comunidad concreta. Esta propuesta afecta a todos los sectores implicados en el contexto educativo (alumnado, profesorado, administración, etc.) y toma como referencia un centro de características singulares, por tratarse de uno de los centros educativos españoles que imparte la enseñanza especializada de idiomas, que se caracteriza por tener un gran número de alumnos, un grupo considerable de profesores y en definitiva tiene una función social muy importante que desempeñar ante la creciente demanda por parte de la sociedad para formar a las personas adultas en el dominio de una o varias lenguas extranjeras.
\end{abstract}

Palabras claves: Compartir información, construcción de conocimiento, software social, Web 2.0

\begin{abstract}
:
Nowadays the spirit of Web 2.0, whereby users become collaborative producers as well as consumers of information, has pervaded all the spheres of people's everyday lives, and the education context could be no exception. This paper puts forward a proposal to make the most of some of the tools which come with Web 2.0 so that all the members of a teaching institution can feel really involved in it and have the feeling of belonging to it. This is a very specific proposal which affects all the actors involved in the teaching context - students, teachers, management, etc. - taking as reference a school with very special characteristics, as it is one the institutions in Spain devoted to the teaching of foreign languages. It has a large number of students, a considerable number of teachers and serves an important social purpose due to the increasing demand of adult students to command one or more foreign languages.
\end{abstract}

Keywords: Information sharing, knowledge building, social software, Web 2.0

\footnotetext{
${ }^{1}$ Escuela Oficial de Idiomas de Burgos (España); juancarlos.araujo@yahoo.es, jcaraujo@educa.jcyl.es
} 


\section{Propuesta para un centro educativo 2.0}

\section{Introducción}

Actualmente se añade la etiqueta «2.0» a diversos conceptos para sugerir que una actividad ha adoptado un nuevo conjunto de herramientas que la aproximan a la idea de Web 2.0 (Crook, 2008). Esta permite nuevos modos de actuación, especialmente nuevas formas de participación social en la red. El concepto de Web 2.0 abarca un amplio conjunto de significados que ponen el acento en el contenido creado por los usuarios, en compartir tanto datos como contenidos y en un esfuerzo colaborativo, así como en la utilización de diversos tipos de software social, nuevos modos de interacción a través de herramientas que se encuentran en la red, y el uso de esta como una plataforma para generar contenidos, modificar su finalidad y consumirlos (Franklin y van Harmelen, 2007).

En las últimas décadas la forma en que se utiliza Internet ha cambiado desde un punto de vista cualitativo (Warschaver y Grimes, 2007). La red permite a los usuarios adquirir un papel más creativo en el proceso de facilitar información y a la hora de construir conocimiento, lo que se ha visto favorecido por el destacado aumento del número de usuarios y de su nivel de implicación en la red. Gracias a la Web 2.0 algunas de las herramientas que la acompañan se han socializado y han adquirido nuevos formatos, por ejemplo, los blogs. La Web 2.0 utiliza las tecnologías que vienen con ella sobre todo para procesos de comunicación y transferencia de conocimiento (Rollett et al., 2007).

Se incorpora a los usuarios en el proceso de creación de contenidos y de ese modo se añade valor al proceso y a sus resultados. Son los usuarios los que modifican la forma de utilizar Internet, al pasar de ser meros espectadores a participar de forma activa en su desarrollo. De este modo, la red evoluciona de un espacio exclusivamente de lectura a serlo de lectura y escritura (De la Torre, 2006). De esa forma, en la actualidad el usuario es el protagonista principal, generando y compartiendo contenidos (Cuesta Morales y Gómez Rodríguez, 2008). Y esta creación de contenidos no significa que obligatoriamente haya que continuar con la idea tradicional respecto al concepto de autor. Un elevado porcentaje de los usuarios serán visitantes o lurkers (Preece, 2000), es decir, personas que tan solo visitan las páginas web pero no aportan contenidos. Dentro del espíritu de la Web 2.0, los usuarios que aportan datos o contenidos esperan ser tratados con respeto y confían que estos estén disponibles para todo el mundo. Asimismo se está 
permitiendo la incorporación de puntos de vista y opiniones de otras personas, lo que lleva a la construcción de conocimiento dentro de un nuevo marco (Pegrum, 2009).

La Web 2.0 se basa en la creación de conocimiento compartido, construido en comunidad (Herrera Jiménez, 2007). Ofrece el potencial de complementar, aumentar y añadir nuevas dimensiones colaborativas al aula y por extensión a todo un centro educativo. Se ha bautizado a las tecnologías de la Web 2.0 "ssoftware socialı porque se considera que consiguen que sea posible estar especialmente conectados, lo que permite a los usuarios desarrollar contenido web de forma colaborativa y que este sea accesible al público en general (Alexander, 2006). La Web 2.0 no es solo un conjunto de aplicaciones, de servicios accesibles en línea, sino que es una actitud con la que estar en la red y compartir el conocimiento (AulaBlog, 2008).

O'Reilly (2005) concibe la Web 2.0 como una plataforma estratégica en la que los datos son introducidos por los usuarios y esto lleva a una inteligencia colectiva. Puede, por tanto, considerarse un entorno orientado al conocimiento donde los usuarios crean contenido manipulable de forma colaborativa, bien sea de forma síncrona o asíncrona, y este se distribuye a tecnologías fijas y móviles. Obtener recursos en Internet es relativamente fácil, puesto que la tecnología permite el acceso a la información a todo el mundo, pero encontrar los recursos adecuados puede suponer un reto.

En la Web 1.0 tan solo unas pocas personas creaban información para un gran número de usuarios, que eran en su mayor parte pasivos (Franklin y van Harmelen, 2007). Por el contrario, la Web 2.0 está integrada por un conjunto de tecnologías sociales de nueva generación cuyos usuarios participan de forma activa para comunicarse y colaboran entre sí mientras establecen conexiones y contactos a lo largo del planeta, a la vez que negocian sus identidades en línea durante este proceso (Pegrum, 2009). Los usuarios utilizan la Web 2.0 como una plataforma donde generar, reutilizar y consumir el contenido que comparten. De ese modo también se convierte en una plataforma de software social que permite a grupos de usuarios establecer relaciones sociales, colaborar y trabajar de forma conjunta (Franklin y van Harmelen, 2007).

Haciendo referencia a algunas de estas herramientas concretas, muchas 


\section{Propuesta para un centro educativo 2.0}

personas alaban las ventajas de los blogs puesto que proporcionan una estructura sencilla que muchos encuentran intuitiva y fácil de utilizar (Rollett et al., 2007). Los docentes pueden usar un blog para publicar anuncios y noticias sobre el curso así como para facilitar retroalimentación a los alumnos. Se pueden emplear con tecnologías de subscripción de contenidos para permitir que los usuarios, de una forma sencilla, puedan estar al tanto de las novedades que se publiquen (Franklin y van Harmelen, 2007). Algunas instituciones, por ejemplo la Universidad de Warwick, hace un gran uso de esta herramienta. De hecho, hay muchos argumentos a favor de su utilización por parte de una institución para diferentes finalidades, entre las que se puede destacar su uso como un recurso pedagógico más en el proceso de enseñanza-aprendizaje, así como en el campo de la investigación y en los aspectos administrativos (Franklin y van Harmelen, 2007). Warschaver y Grimes (2007) hablan de otro tipo de blog que busca proporcionar información y comentarios $u$ observaciones respecto a un tema, materia, proyecto o producto. Lo llaman blog del conocimiento o k-log (knowledge log). También señalan que muchos blogs permiten que exista una cierta interactividad entre la audiencia y el autor. Bloch (2007), por su parte, llegó a la conclusión de que participar en un blog debería permitir a los alumnos convertirse en colaboradores y no simplemente en consumidores de información que hallan en Internet.

Un wiki es una página web colaborativa cuyo contenido pueden editar sus visitantes, que permite a los usuarios crear y editar páginas web de forma colaborativa (Chao, 2007). Entre los posibles usos pedagógicos que se podrían dar a los wikis, Duffy y Bruns (2006), señalan que se puede emplear para publicar o alojar recursos como la programación didáctica y materiales imprimibles del curso. Los alumnos pueden editar estos recursos y realizar comentarios que todo el mundo puede ver.

Tanto Augar et al. (2004) como Naish (2006) demuestran que los wikis se pueden emplear para aumentar la interacción social en línea entre los alumnos a la vez que para distribuir contenido entre el conjunto de estudiantes, para elaborar repositorios de información y para la producción de documentos de forma colaborativa. También se pueden emplear para la creación de listas de lecturas recomendadas que incluyan comentarios (Franklin y van Harmelen, 2007).

Lamb (2004), por su parte, describe varios usos que se pueden dar a los wikis 
fuera del aula. Los centros o agencias de colocación pueden utilizar wikis para alojar y organizar ofertas laborales. De igual modo, las universidades pueden usarlos como un medio que permita a los alumnos expresar su opinión respecto a las distintas políticas y medidas que la universidad defiende y pone en práctica.

A diferencia de lo que sucede en los blogs, donde el autor suele ser habitualmente una sola persona, en un wiki hay diversos autores y todos ellos pueden crear contenidos nuevos y/o editar los publicados por el resto de participantes en el wiki, lo que hace que se pase de una autoría individual, más característica de un blog, a una autoría colaborativa y colectiva, donde no se sabe realmente quién es el autor, puesto que lo son todas las personas que han participado en su elaboración.

Parker y Chao (2007) confirman esto y añaden que en los wikis la información se estructura en temas mientras que en los blogs aparecen en orden cronológico inverso. Es por eso por lo que se espera que los temas que se tratan en los wikis evolucionen y se expandan hasta convertirse en una base permanente de conocimiento, mientras que el orden cronológico inverso de los blogs hace que resulte difícil encontrar todas las entradas relacionadas con un mismo tema 0 navegar por todas las entradas referidas a ese asunto. Los wikis, por su parte, muestran qué información está relacionada y hacen que resulte fácil navegar por ella (Woolf, 2006).

\section{Descripción del centro educativo para el que se presenta la propuesta}

El centro educativo que se escoge como referencia para la propuesta que se describe a continuación es la Escuela Oficial de Idiomas (EOI) de Burgos, España, que es una de las mayores de la Comunidad Autónoma de Castilla y León, España, en cuanto a número de alumnos y profesores. En la misma se ofertan los idiomas alemán, español, francés, inglés, italiano y ruso. De todos ellos se imparten los niveles básico, intermedio y avanzado, a excepción de ruso, del que tan solo se ofertan los niveles básico e intermedio. Asimismo, en el caso de inglés, y al igual que sucede en las EE.OO.II. de las capitales de provincia y en alguna más, desde el curso 2010-2011 también se imparte el nivel Cl del Marco Común Europeo de Referencia (MCER) para las lenguas. Además, este centro cuenta con una sección de EOI en la localidad de Briviesca, donde se oferta el idioma inglés en todos los cursos y niveles, 
a excepción del nivel Cl.

Las Escuelas Oficiales de Idiomas (EE.OO.II.) son los centros educativos públicos españoles que imparten enseñanzas especializadas de idiomas que «tienen por objeto capacitar al alumnado para el uso adecuado de los diferentes idiomas, fuera de las etapas ordinarias del sistema educativo, y se organizan en los niveles siguientes: básico, intermedio y avanzado. (...) Para acceder a las enseñanzas de idiomas será requisito imprescindible tener dieciséis años cumplidos en el año en que se comiencen los estudios. Podrán acceder asimismo los mayores de catorce años para seguir las enseñanzas de un idioma distinto del cursado en la educación secundaria obligatoria. (...) [Estos centros] fomentarán especialmente el estudio de las lenguas oficiales de los Estados miembros de la Unión Europea, de las lenguas cooficiales existentes en España y del español como lengua extranjera. Asimismo, se facilitará el estudio de otras lenguas que por razones culturales, sociales o económicas presenten un interés especialı) (Ley Orgánica 2/2006, artículos 59 y 60).

Por su parte la Ley Orgánica 8/2013 establece en el artículo 47 que «las Enseñanzas [especializadas] de Idiomas [que se imparten en las Escuelas Oficiales de Idiomas] tienen por objeto capacitar al alumnado para el uso adecuado de los diferentes idiomas, fuera de las etapas ordinarias del sistema educativo, y se organizan en los niveles siguientes: básico, intermedio y avanzado. Estos niveles se corresponderán, respectivamente, con los niveles A, B y C del Marco Común Europeo de Referencia para las Lenguas, que se subdividen en los niveles A1, A2, B1, B2, C1 y C2») (p. 978933).

Hay una Escuela Oficial de Idiomas (EOI) en cada una de las capitales de provincia españolas, así como en las principales localidades de las provincias. Asimismo, algunas EE.OO.Il cuentan con una extensión o sección en poblaciones cercanas, por lo que en la actualidad hay más de 300 centros donde se imparten estas enseñanzas especializadas entre EE.OO.II. y extensiones o secciones repartidas a lo largo de España. En todas ellas se imparte el idioma inglés, y en la inmensa mayoría también francés. La oferta del resto de idiomas se establece en función del tamaño de la localidad donde se haya ubicado el centro y de razones culturales, sociales o económicas.

El alumnado de estos centros educativos es en su mayor parte personas adultas, de al menos dieciséis años de edad, que suelen ser mayoritariamente 
estudiantes universitarios o trabajadores en activo, o en los últimos tiempos, en situación de desempleo. Dado el número de obligaciones y compromisos de todo tipo a los que tienen que hacer frente (personales, académicos, laborales, familiares, etc.), en ocasiones no acuden de forma habitual a clase, ni disponen del tiempo necesario para realizar las diferentes tareas que se les solicita hacer fuera de clase.

El Departamento de Inglés está integrado por 24 personas, habiendo tenido una plantilla de 27 profesores hasta el curso 2009-2010. En la Sección de EOI de Briviesca hay una plaza de plantilla y media plaza. Con las medias jornadas, en algún momento el Departamento ha llegado a estar formado por un total de 32 personas. Los restantes departamentos tienen la siguiente plantilla: alemán (cuatro en plantilla y media plaza), español (uno en plantilla y tres cuartos de plaza), francés (seis en plantilla y media plaza), italiano (dos en plantilla y media plaza) y ruso (una plaza).

La Escuela oferta los siguientes horarios. Por la mañana de 9:30 a 11:30, donde el alumnado lo integran principalmente jubilados, amas de casa, estudiantes universitarios y en los últimos años desempleados. Por la tarde el horario de clases es de 16:00 a 21:00 horas. De 16:00 a 19:00 horas predominan los alumnos adolescentes. También hay amas de casas y funcionarios. A partir de las 19:00 horas el alumnado se compone mayoritariamente de trabajadores en activo y estudiantes universitarios.

Existen diferentes modalidades de enseñanza. La enseñanza presencial u oficial, donde los alumnos asisten a clase de forma habitual. Hay alrededor de 4.000 alumnos que cursan esta modalidad de enseñanza entre los seis idiomas que se ofertan. Enseñanza libre, donde los alumnos no asisten a clase y tan solo tienen derecho a presentarse a la realización de las pruebas de certificación, que les permite obtener una titulación que acredita que poseen un nivel concreto de dominio del idioma extranjero en relación con el MCER: el nivel A2 si superan las pruebas del Nivel Básico, B1 las del Nivel Intermedio, B2 del Avanzado y finalmente Cl para el idioma inglés. Normalmente suele haber en torno a 350 alumnos matriculados en esta modalidad de enseñanza.

En el caso de los alumnos libres, y especialmente de inglés, muchos son estudiantes universitarios que disfrutan de una beca ERASMUS y al finalizar el curso 


\section{Propuesta para un centro educativo 2.0}

académico se examinan del idioma del país en el que han realizado sus estudios universitarios. También hay un número importante de personas de la ciudad, que por diversos motivos, principalmente laborales, no residen de forma habitual en la misma, pero que en vez de examinarse por libre en la localidad donde suelen vivir, o en una cercana, prefieren hacerlo en esta EOI. Todos estos alumnos solicitan de forma continuada información sobre las fechas y organización de los exámenes libres.

Por último, en el caso de inglés, se oferta la modalidad a distancia, a través del curso That's English, para los niveles básico e intermedio, y estos alumnos cuentan con una tutoría presencial semanal. Si superan los módulos correspondientes a cada nivel, tienen que presentarse a la misma prueba de certificación que realizan los alumnos oficiales y libres de los niveles básico e intermedio.

Dado el elevado número de integrantes del Departamento de Inglés, a la hora de elaborar la programación didáctica de cada nivel y curso, exámenes, etc., están divididos en varias comisiones por nivel y curso. De esa forma, cuando no hay reunión de Departamento se celebran reuniones de estas comisiones, principalmente para la elaboración y revisión de las diferentes pruebas y exámenes que se celebran a lo largo del curso, así como para la creación de material extra que poder utilizar en clase. Dado que no todos los profesores que imparten un curso forman parte de la comisión correspondiente, hay momentos a lo largo del curso en los que esas personas tienen que hacer llegar a la comisión opiniones sobre el material o exámenes elaborados, sugerencias de mejora, propuestas de cambio, etc.

El centro dispone de una página web donde se incluye la información que se considera más relevante para los alumnos, así como el acceso al portal del alumno, donde los estudiantes pueden consultar el número de faltas de asistencia, las calificaciones, su expediente académico, etc.

\section{Propuesta}

El proyecto para la inclusión de herramientas de la Web 2.0 busca su implantación y utilización a todos los niveles: administrativo, organizativo y académico, para un mejor y más coordinado funcionamiento de todo lo que afecta al día a día de un 
centro educativo de sus características.

A nivel administrativo, además de la página web, se propone crear un blog en el que los nuevos alumnos pudieran plantear sus dudas, preguntas, etc. respecto al funcionamiento de la Escuela, para que se les aclararan por parte del centro, pero deberían hacerlo los antiguos alumnos, que en su momento tuvieron el mismo tipo de dudas o preguntas que puedan tener esos nuevos alumnos.

Lógicamente es importante que aparezca toda la información y documentación relevante en lo referente a cuestiones administrativas, incluyendo documentos que respondan a las preguntas más frecuentes. Esto es algo que ya se hace en la página web del centro en muchas ocasiones, pero parece más conveniente que en vez de la página web se utilizara un blog para ello, puesto que muchas veces los alumnos plantean el mismo tipo de cuestiones y dudas una y otra vez, y hacerlo mediante un blog, en el que los alumnos formulen sus preguntas y planteen sus dudas a través de los comentarios, permite que esa información quede ahí de forma permanente, y con casi toda seguridad completamente aclarada, para así evitar tener que facilitar la misma información reiteradamente. Nuevamente, deberían ser los mismos alumnos los que proporcionaran esa información, en vez del centro, siempre y cuando la misma fuese correcta, para lo que se precisa la supervisión de esos comentarios por parte del personal administrativo del centro. Para ayudar a los usuarios o visitantes de la página web estar al corriente de las nuevas entradas y comentarios que se publiquen convendría incluir RSS feeds.

La información más importante de carácter administrativo también debe comunicarse en las redes sociales que cuentan con un mayor número de usuarios: Facebook, Twitter, Google +, etc. Información relativa a los plazos de matrícula tanto oficial como libre, para la preinscripción para el curso siguiente, las fechas de los exámenes, etc. deben anunciarse en estas redes sociales con los enlaces correspondientes a la sección pertinente de la página web del centro y demás herramientas de la Web 2.0 que se estén utilizando para difundir información sobre esos temas.

Por lo que se refiere a un nivel organizativo, se sugiere utilizar wikis en los que se incluyan los temas que se van a tratar en las reuniones de departamento, comisiones y claustros con las propuestas que se van a plantear en las mismas, en 


\section{Propuesta para un centro educativo 2.0}

especial cuando se trata de cuestiones de gran trascendencia (cambios en los formatos de los exámenes, pruebas de nivelación, etc.) para así disponer de tiempo para estudiarlas así como para realizar comentarios y sugerencias, proponer cambios, etc. que todo el mundo pueda leer antes de la celebración de esas reuniones y haber pensado sobre los mismos y de ese modo que las reuniones resulten más rápidas y fluidas. Nuevamente parece conveniente incluir RSS feeds para que se pueda comunicar a los integrantes del wiki las modificaciones, cambios, etc. que se vayan produciendo.

Esto se percibe como especialmente útil en el caso de las reuniones de las comisiones por nivel y curso cuando se va a revisar la propuesta de exámenes, y especialmente en el caso de los profesores que imparten dicho curso pero que no forman parte de esa comisión. Este tipo de reuniones suelen ser largas porque hay mucho material que revisar. Si parte de ese trabajo ya se ha hecho de antemano a través de un wiki, las reuniones pueden resultar más productivas y permitirían centrarse más en aquellos aspectos que puedan ser o parecer más problemáticos o conflictivos.

Asimismo parece muy práctico a la hora de elegir nuevos métodos o libros de texto, de tal forma que después de examinar los métodos que haya para un determinado nivel y curso, cada uno indicara los aspectos positivos y negativos, que en su opinión, tiene cada uno, para que de ese modo todo el mundo pudiese hacerse una mejor idea del método que puede resultar más adecuado. En muchas ocasiones a la hora de realizar esta tarea no se sabe muy bien por donde empezar. A partir de la opinión de otros compañeros, se puede comprobar si se está de acuerdo con lo que indican sobre cada libro de texto, y al mismo tiempo se propicia que no sean tan solo los miembros de la comisión los que participen en la realización de una propuesta para ese nivel y curso, como suele ser lo habitual en la actualidad, sino que se permite que lo haga cualquier integrante del Departamento.

También sirve para comprobar si se puede establecer una buena progresión entre un curso y el siguiente dentro del mismo nivel, o entre un nivel y otro con los libros de texto que se propongan. En muchas ocasiones no se ha escogido bien un método o libro de texto para un curso o nivel concreto precisamente por no disponer de dicha información. 
De igual modo, se pueden utilizar herramientas como Google Docs o wikis privados para la elaboración de los materiales para las diferentes pruebas y exámenes que se realicen a lo largo del curso, así como material extra para utilizar en clase, y de ese modo se puede colaborar y compartir material y trabajo con profesores de otras EE.OO.II. de la región y de otras comunidades autónomas. Asimismo con herramientas como Google Sites, se puede crear un banco de exámenes y ejercicios de práctica, lo que además de permitir compartir material, trabajo e ideas, supone una disminución del trabajo que se tiene que realizar en ese sentido para así poder dedicar más tiempo a otros aspectos de la labor docente.

Por lo que se refiere a nivel académico, conviene crear blogs o wikis en los que se incluyan podcasts y vidcasts, bien grabados por los profesores del centro o alojados en YouTube, que traten aspectos que hay que repetir año tras año, y en todos los niveles y cursos. Por ejemplo, en el caso del inglés, serían aspectos como la pronunciación de la desinencia verbal-ed de los pasados y participios de los verbos regulares; la terminación -s de la tercera persona del singular del presente simple, el plural de los sustantivos y el caso posesivo; la pronunciación de los fonemas ingleses; los símbolos fonéticos; la pronunciación de los verbos irregulares, etc. Estos blogs o wikis pueden ser utilizados por todos los profesores, a los que pueden enlazar desde sus blogs personales o de aula. También se podría enlazar a ellos desde las páginas de cada departamento del mismo modo que se hace a enlaces de interés externos a la Escuela.

De igual modo, se debería elaborar unos blogs por nivel y curso en los que se incluyeran actividades y materiales que puedan resultar de interés y ayuda para los alumnos de esos cursos, y a los que nuevamente se podría enlazar desde los blogs de los profesores o de aula.

Asimismo, convendría crear un blog, o serie de blogs si se prefiere hacer por idioma, nivel y curso, en el que se sugiera a los alumnos estrategias y materiales que pueden utilizar en su proceso de aprendizaje, de tal modo que sean ellos los que, a través de los comentarios que publiquen, puedan establecer o comparar la eficacia de los diferentes recursos que se les facilita o bien sugiere que empleen, o que ellos utilizan en su proceso de aprendizaje de una lengua extranjera. De ese modo se produciría una colaboración entre los alumnos. Asimismo se estaría enseñando a los alumnos estrategias de aprendizaje, y ellos serían conscientes de 


\section{Propuesta para un centro educativo 2.0}

ello y reflexionarían sobre esto, y se estaría apuntando a diferentes estilos de aprendizaje. Una vez iniciado, este proceso se podría trasladar a un trabajo a través de un wiki, si se percibe que hay alumnos que muestran interés por el tema y hay profesores dispuestos a encargarse de la organización y gestión del mismo.

En los blogs de aula, además de utilizarlos para informar sobre la tarea para casa, se pueden incluir las actividades realizadas en clase, así como los materiales utilizados, si los mismos son auténticos y se han encontrado en la red y no hay problemas en lo que se refiere a los derechos de autor, algo que sí que sucedería en el caso de materiales elaborados para los métodos y libros de texto que se usan en las aulas. De ese modo, los alumnos que no hayan podido asistir a clase tendrían la posibilidad de realizar las mismas actividades que los que sí que lo hicieron, y tanto unos como otros las podrían repetir cuantas veces desearan. En esos mismos blogs se pueden plantear discusiones o tareas de extensión o refuerzo de lo tratado en clase, de modo que sirvan de práctica adicional y voluntaria para los alumnos.

Los ejercicios de expresión escrita, que representan una gran dificultad para los alumnos con independencia de su edad y nivel, pueden realizarse a través de un wiki. Dadas las características del alumnado que asiste a las EE.OO.Il., que normalmente no dispone de mucho tiempo para dedicar al trabajo personal e individual, y dada la dificultad que les supone este tipo de ejercicios, cuando se solicita un trabajo de expresión escrita de práctica un número importante de alumnos no lo realiza. Sin embargo, desarrollarlo a través de un wiki, y dado su carácter de trabajo colaborativo, puede conseguir que los alumnos perciban la tarea como más asequible y haya más gente que se anime a realizarla, o al menos a participar en alguna fase del proceso de escritura y posterior revisión y análisis, tal y como se ha demostrado que sucede en otros casos en los que se busca involucrar a los alumnos en los procesos previos y posteriores a los de redacción del ejercicio.

Por último, y utilizando alguna o varias de las herramientas de la web 2.0, se puede animar a los alumnos a que elaboren proyectos, trabajos, presentaciones, etc. para su posterior publicación en repositorios como YouTube, Scribd o Slideshare, etc. haciendo de ese modo que la actividad sea más motivadora, al mismo tiempo que los alumnos la perciban como real y cercana. A la hora de realizar este tipo de actividades se puede intentar buscar la ayuda y colaboración de hablantes nativos o de otros alumnos de la lengua meta que se encuentren en otros países. 
Finalmente, y por medio de wikis públicos se sugiere crear una especie de club de lectura en línea, partiendo de los libros que cada departamento recomienda leer para cada curso y nivel. En muchas ocasiones los alumnos piden consejo respecto a qué libros, revistas, películas podrían leer o ver. Mediante esta opción serían los propios alumnos los que se encargaran de recomendar a otros compañeros lo que podrían leer o ver, y se pueden establecer debates que ayuden a una mejor comprensión de una obra o película concreta, o a que estas se consideren desde diferentes puntos de vista. De igual modo se facilitan información sobre ellas, y si se hace en la lengua meta, se practica esta en un contexto de comunicación auténtico, en el que, al tratarse de un wiki público, cualquier persona en cualquier punto del planeta puede participar.

\section{Conclusiones}

En este artículo se ha querido mostrar como el espíritu de la Web 2.0, algo que cada vez es más habitual en la vida cotidiana de las personas, puede aplicarse en los procesos de enseñanza-aprendizaje así como en aquellos centros e instituciones donde estos se llevan a cabo. De ese modo se estará sacando partido al gran potencial del software social que acompaña a la Web 2.0, y se permitirá a los usuarios de estos centros adquirir un papel protagonista que ya han alcanzado en otros muchísimos ámbitos de la vida y en Internet. Eso también ayudará a afianzar y consolidar el sentimiento de pertenencia de esos usuarios, en su mayor parte, alumnos, a una comunidad concreta. Sin embargo, no se limita tan sólo al alumnado, sino que se plantea para todas las personas que por motivos académicos o profesionales forman parte de dicho centro (profesorado y personal administrativo).

Para ello se realiza una propuesta tomando como referencia un centro educativo muy concreto, que imparte unas enseñanzas muy específicas, que tiene un elevado número de alumnos de diferentes edades, intereses, nacionalidades, niveles socioculturales, económicos, a la vez que cuenta con un considerable número de profesores que desarrollan su labor en esta institución educativa, que tiene unas características muy específicas por el tipo de enseñanzas que imparte y las características del alumnado que acude a ella. Lógicamente esta propuesta puede servir de modelo o punto de partida para dar respuesta a las necesidades 
concretas de cada centro o institución educativa.

\section{Referencias bibliográficas}

ALEXANDER, B. (2006). Web 2.0: A new wave of innovation for teaching and learning? Educause Review, $41(2)$ Recuperado de http://www.educause.edu/ir/library/pdf/ERM0621.pdf

AUGAR, N., RAITMAN, R., y ZHOU, W. (2004). Teaching and learning online with wikis. Proceedings of the 21st Australasian Society for Computers in Learning in Tertiary Education (ASCILITE) Conference, Perth: December 5-8, 95-104. Recuperado de http://www.ascilite.org.au/conferences/perth04/procs/pdf/augar.pdf

Aulablog. (2008) Web 2.0: más allá de una realidad tecnológica al servicio de la educación. Espacios de Reflexión, Primer Congreso Nacional de Internet en el Aula, 2008. Recuperado de http://www.slideshare.net/aulablog/web-20352227

BLOCH, J. (2007). Abdullah's blogging: A generation 1.5 student enters the blogosphere. Language Learning \& Technology, 11 (2), 128-141.

BYRON, M. (2005). Teaching with Tiki. Teaching Philosophy, 28(2), 105-113.

CHAO, J. (2007). Student project collaboration using Wikis. En Proceedings of the 20th Conference on Software Engineering Education \& Training (pp. 255-261). IEEE Computer Society.

CROOK, C. (2008). What are web 2.0 technologies, and why do they matter? En N. Selwyn, (Ed.), Education 2.0?: designing the web for teaching and learning: a commentary, (pp. 6-9). Teaching and Learning Research Programme. Recuperado de http://eprints.ioe.ac.uk/6217/1/Selwyn2008education.pdf

CUESTA MORALES, P., y GÓMEZ RODRÍGUEZ, A. M. (2008). Web 2.0 e educación. Revista de Formación e Innovación Educativa Universitaria (REFIEDU), 1 (2), 5257.

DAVIES, W. (2003). You don't know me but ... Social capital and social software. London: The Work Foundation. Recuperado de http://www.theworkfoundation.com/Assets/PDFs/you dontknowme.pdf

DE LA TORRE, A. (20016). Web Educativa 2.0. Edutec-e, Revista Electrónica de Tecnología Educativa, 20.Recuperado 
http://www.vib.es/depart/gte/gte/edutec-e/revelec20/anibal20.htm

DUFFY, P., y BRUNS, A. (2006). The use of blogs, wikis and RSS in education: A conversation of possibilities. Proceedings of the Online Learning and Teaching Conference 2006, Brisbane: September 26. Recuperado de https://olt.qut.edu.au/udf/OLT2006/gen/static/papers/Duffy OLT2006_paper.p df

FRANKLIN, T., y VAN HARMELEN, M. (2007). Web 2.0 for content for learning and teaching in higher education. JISC. Recuperado de www.jisc.ac.uk/media/documents/programmes/digitalrepositories/web2contentlearningand-teaching.pdf

HERRERA JIMÉNEZ, F. J. (2007, Invierno). Web 2.0 y didáctica de las lenguas. Glosas didácticas: revista electrónica internacional de didáctica de las lengua y sus culturas, $16 . \quad$ Recuperado de http://www.um.es/glosasdidacticas/gdl6/02herrera.pdf

LAMB, B. (2004). Wide open spaces: Wikis, ready or not. EDUCAUSE Review, 39(5) (September/October), 36-48. Recuperado de http://www.educause.edu/pub/er/erm04/erm0452.asp?.bhcp=1

LEY ORGÁNICA 2/2006, de 3 de mayo, de Educación. BOLETíN OFICIAL DEL ESTADO, $\mathrm{n}^{\circ} 106$, de 4 de mayo de 2006, 17158-17207.

LEY ORGÁNICA 8/2013, de 9 de diciembre, para la mejora de la calidad educativa. BOLETíN OFICIAL DEL ESTADO, n²95, de 10 de diciembre de 2013, 9785897921.

NAISH, R. (2006). Can wikis be useful for learning? e.learning Age. Recuperado de http://www.qiconcepts.co.uk/pdf/Can\%20Wikis\%20be\%20useful\%20for\%20lear ning.pdf

O'REILLY, T. (2005). What is web 2.0: Design patterns and business models for the next generation of software. Recuperado de http://www.oreillynet.com/pub/a/oreilly/tim/news/2005/09/30/what-is-web20.html

PARKER, K., y CHAO, J. (2007). Wiki as a teaching tool. Interdisciplinary Journal of elearning and Learning Objects, 3(1), 57-72.

PEGRUM, M. (2009). Communicative networking and linguistic mashups on Web 2.0. En M, Thomas (Ed.), 2009, Handbook of research on Web 2.0 and second 
language learning, (pp. 20-41). USA; IGI Global.

PREECE, J. (2000) Online Communities: Designing Usability and Supporting Sociability. Nueva York, NY: John Wiley \& Sons.

ROLLETT, H., LUX, M., STROHMAIER, M., y DOSINGER, G. (2007). The web 2.0 way of learning with technologies. International Journal of Learning Technology, 3(1), 87-107.

VALLANCE, M., VALLANCE, K., y MATSUI, M. (2009). Communicative networking and linguistic mashups on Web 2.0. En M. Thomas (Ed.), Handbook of research on web 2.0 and second language learning, pp. 1-19. Hershey PA: IGI Global.

WARSCHAUER, M., y GRIMES, D. (2007). Audience, authorship, and artifact: the emergent semiotics of web 2.0. Annual Review of Applied Linguistics, 27(1), 123.

WOOLF, B. (2006). Wiki vs. Blog. IBM DEVELOPERWORKS. Recuperado de http://www03.ibm.com/developerworks/wikis/display/woolf/Wiki+vs.+Blog.

\section{Cómo citar este articulo:}

Araujo Protugal, Juan C. (2016). Propuesta para un centro educativo 2.0 EDEMTIC, Revista de Educación Mediática y TIC, 5(1), 117-133. 